ation of facilities for isolation (hospital, residential, other) and mechanisms of enforcement. The guidelines should be flexible and allow state or local officials to use their knowledge of local circumstances and judgment to determine which measures are most applicable. Successful implementation of containment measures will depend on public trust and require a consistent and clear communications plan. Groups that will be instrumental in implementing an effective response, such as the transportation industry, law enforcement, emergency services, and federal, state, and local legal experts, should be engaged early in the planning process. Training modules and drills that utilize realistic scenarios to evaluate the decisionmaking process and assess the feasibility of implementing containment measures should be developed, tested, and disseminated.

For healthcare preparedness, key considerations include defining infection control precautions for evaluating and handling patients with respiratory illness in the outpatient and inpatient setting, educating and training clinicians on clinical features of SARS and appropriate use of personal protective equipment, and building strong partnerships and collaborations between the clinical and public health communities, including cross-training staff in the areas of infection control and public health. Furthermore, issues of resource allocation and surge capacity in the event of a major SARS epidemic should be addressed.

For laboratory preparedness, guidelines should be updated for specimen collection, transport, and storage and the appropriate use of diagnostic tests and interpretation of test results. Surge capacity for testing at the federal, state, and local levels should be identified, and an adequate supply of reagents that have been properly validated and checked for quality should be ensured. While research to develop second-generation assays for improved diagnosis of SARS-CoV infection should continue, efforts should also focus on improving the performance of existing assays. Biosafety recommendations for specimen collection and laboratory processing must be updated. Guidelines for environmental testing for SARS-CoV must be developed and should include information on the role and utility of testing.

For communications and education, messages and curricula should be developed that target three audiences: public (including policy mak- ers), physicians, and public health workers. Materials that were developed in response to the SARS outbreak must be reviewed and updated. Education and training efforts should focus on key areas, such as recognizing the clinical manifestations of SARS, appropriate use of infection control practices and personal protective equipment, rationale and practical guidance for implementing isolation and quarantine, and appropriate use and interpretation of laboratory diagnostic tests.

The information and ideas shared in this meeting are helping the public health and healthcare communities define priority SARS preparedness activities at the national, state, and local levels.

\section{Umesh D. Parashar* and Larry J. Anderson*}

${ }^{*}$ Centers for Disease Control and Prevention, Atlanta, Georgia, USA

Address for correspondence: Umesh D. Parashar, Division of Viral and Rickettsial Diseases, National Center for Infectious Diseases, Centers for Disease Control and Prevention, 1600 Clifton Rd, NE, Atlanta, Georgia 30333, USA; fax: 404-639-4960; email: uap2@cdc.gov

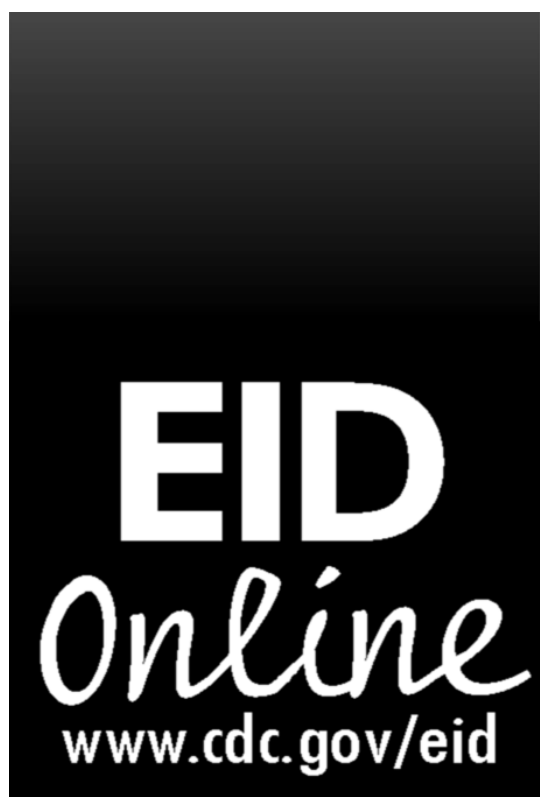

\title{
Correction, Vol. 10, No. 1
}

In the article "Bacillus anthracis Incident, Kameido, Tokyo, 1993" by Hiroshi Takahashi, et al., errors occurred in the 4th paragraph under "Discussion" on page 119: mu symbols were inadvertently replaced by the letter "m." The corrected sentences appear below:

The human respiratory infectious dose 50 (dose that will produce an infection in $50 \%$ of exposed persons) is unknown but has been estimated to be 8,000 to 10,000 spore-bearing particles $<5 \mu \mathrm{m}$ in diameter (7). Kameido residents described a gelatinous substance, suggesting the suspension would be poorly dispersed and droplets would be too large to form particles $<5 \mu \mathrm{m}$ in diameter.

In addition, the name of the lead author of this article is misspelled in the table of contents of this issue. In the table of contents, the article should be attributed to "H. Takahashi et al."

The corrected article appears online at http://www.cdc.gov/ncidod/EID/ vol10no1/03-0238.htm

We regret any confusion these errors may have caused. 